\title{
Globe
}

Revue internationale d'études québécoises

\section{Le jeu des espaces publics dans Jésus de Montréal de Denys}

Arcand

\section{The play of public spaces in Jésus de Montréal by Denys Arcand}

\section{Tony Simons}

Volume 2, numéro 2, 1999

URI : https://id.erudit.org/iderudit/1000473ar

DOI : https://doi.org/10.7202/1000473ar

Aller au sommaire du numéro

Éditeur(s)

Globe, Revue internationale d'études québécoises

ISSN

1481-5869 (imprimé)

1923-8231 (numérique)

Découvrir la revue

Citer cet article

Simons, T. (1999). Le jeu des espaces publics dans Jésus de Montréal de Denys Arcand. Globe, 2(2), 147-160. https://doi.org/10.7202/1000473ar
Résumé de l'article

Cet article explore la manière dont le film Jésus de Montréal de Denys Arcand propose un discours dans lequel les espaces soulèvent des questions d'identité personnelle et de liberté d'expression. Ce jeu d'espaces, basé sur la notion de la perception, permet de dévoiler la localisation du pouvoir, qui coïncide avec des débats autour des espaces-identité et des espaces-territoire au Québec. Ces débats touchent non seulement le Québec et le Canada, mais aussi les peuples du monde entier. 


\section{Le jeu des espaces publics dans Jésus de Montréal de Denys Arcand}

\section{Tony Simons* \\ Université de Reading (Royaume-Uni)}

Réalisé par Denys Arcand en 1988, le film Jésus de Montréal a obtenu un vaste succès et reste un des films québécois les plus connus auprès du public étranger, notamment européen. De facture symbolique, l'intrigue de ce film propose une réactualisation de la Passion du Christ, dans une mise en scène qui entrecroise de manière subtile les thèmes de la religion catholique et du Christ à la vie du personnage principal, un jeune comédien, Daniel Coulombe, qui accepte de reprendre une pièce sur la Passion montée depuis des années dans les jardins d'un sanctuaire catholique de Montréal, l'oratoire Saint-Joseph. Suivant les instructions du père Leclerc, un prêtre du sanctuaire, il modernise l'action et joue lui-même le rôle du Christ. La pièce connaît un succès retentissant, mais elle se heurte à la désapprobation des autorités catholiques qui n'acceptent pas l'interprétation des textes des Évangiles que Daniel oppose à la leur et qui demandent l'arrêt des représentations. Pourtant, la troupe donne une dernière représentation qui se termine tragiquement au moment où Daniel est blessé sur la croix lors d'une échauffourée provoquée par les gardes de sécurité, puis meurt. D'entrée de jeu, une véritable dialectique s'impose au spectateur, dans un mouvement de balancier qui oppose à la fois le Christ et Daniel, la vie quotidienne au Québec et la représentation des grands mythes occidentaux.

Les scènes principales du film ont été conçues pour suggérer et renforcer chaque fois de manière singulière les parallèles entre la vie de Jésus et celle du personnage principal. Le film, réalisé de manière

\footnotetext{
- Isabelle Décarie a participé à la réécriture de ce texte.
} 


\section{REVUE INTERNATIONALE D'ÉTUDES QUÉBÉCOISES}

linéaire, s'offre comme "une paraphrase de la Passion de Jésus"', ce que suggère lui-même le réalisateur :

J'ai essayé d'écrire comme est écrit l'Évangile selon saint Marc (mon préféré). Dans cet Évangile, il y a un personnage principal qui est entouré de disciples, quatre ou cinq disciples principaux et il va partout. C'est une marche à travers la Galilée. Il rencontre Marie-Madeleine, Marie sa mère, Ponce Pilate, les grands prêtres, jusqu'à ce qu'il meure. C'est un film [...] linéaire mais avec des personnages secondaires qui viennent dire parfois deux ou trois phrases, d'autres qu'on ne voit qu'une fois?.

Avant même la présentation du générique, les thèmes centraux de la Passion - Dieu, la foi, l'amour et la mort - sont annoncés dans un contexte différent de celui de la religion catholique, par le biais d'un extrait des Frères Karamazov que l'on joue au théâtre. À la fin de la représentation, après le suicide de Smerdiakov, Denise Quintal, une productrice d'une grande agence de publicité qui assistait au spectacle, annonce qu'elle "veut la tête du comédien» pour sa campagne de publicité, une allusion à saint Jean-Baptiste, annonciateur de la venue du Christ.

Après le générique, une suite de scènes s'enchaînent pour lier la vie de Jésus à celle de Daniel. Ce dernier part à la recherche de comédiens (et de disciples) et assiste à l'audition d'une publicité pour une marque de bière à la fin de laquelle, en colère, il renverse les tables et l'équipement, un parallèle de l'épisode des marchands du Temple de l'Évangile. Il parait ensuite devant un juge (joué par Arcand lui-même) dont le personnage rappelle Ponce Pilate. Enfin, blessé sur la croix à la fin de la dernière représentation de la Passion sur le mont Royal, il est amené à l'hôpital. Contrairement au Christ, il ne ressuscite pas, mais quand il meurt, ses organes sont donnés à

\footnotetext{
' Gilles Marsolais et Claude Racine, «Entretien avec Denys Arcand à propos de Jésus de Montréals, 24 Images, $n^{\circ} 43$, été 1989, p. 8.

${ }^{2}$ Yves Rousseau, «Entretien avec Denys Arcand», Ciné-Bulles, vol. 8, ${ }^{\circ} 4$, juin-août 1989, p. 5.
} 
d'autres malades et sauvent des vies, ce qui n'est pas sans rappeler le don symbolique du corps du Christ dans l'Eucharistie.

La structure fondatrice du film s'appuie ainsi sur le contraste entre d'un côté le quotidien québécois, la vie à Montréal et de l'autre, le monde des mythes séculaires et les grandes références culturelles occidentales. De la sorte, le film ouvre le champ de son interprétation à tous les publics, non seulement québécois, mais aussi étrangers. Ce portrait clivé entre un monde universel et local se mesure également à travers l'intrigue et les personnages, mais aussi, de manière plus éloquente encore, dans les espaces qui servent de lieux à l'action.

Dans le scénario d'Arcand, la nature de ces espaces est clairement indiquée. Ils sont ou «intérieurs» ou "extérieurs», avec une précision supplémentaire de «jour» ou de «nuit». Ces indications, tout en étant usuelles dans un scénario, renforcent la dialectique du film, ce que Gilles Marsolais avait déjà remarqué :

Globalement, on retrouve dans Jésus de Montréal une structure binaire [...] opposant deux classes sociales et deux modes de vie [...]. Cette structure binaire, et le réseau d'oppositions qu'elle sous-tend, renvoie évidemment à la confrontation des valeurs, spirituelles et matérielles, véhiculées par les acteurs et par les "marchands du Temple» [...]. Aussi, la figure emblématique du Christ fournit à Arcand l'occasion d'explorer de façon dialectique les rapports actuels du jeu et de la vie, de confronter des attitudes de soumission apparente et de domination au sein de la Cité [...].

C'est justement le rôle des espaces au sein de cette structure dédoublée de domination et de soumission qui fera l'objet de cet article. En se servant du jeu des interprétations des espaces, on peut s'interroger non seulement sur les valeurs éternelles présentées dans le film, mais aussi, dans le contexte québécois, sur la politique et l'avenir du pays.

${ }^{3}$ Gilles Marsolais, $\star D u$ spirituel dans l'art», 24 Images, $n^{\circ}$ s 44-45, automne 1989, p. 41. 
Iouri Lotman ${ }^{4}$ propose que toute culture puisse se définir en divisant son territoire en deux, c'est-à-dire en «son propre espace», qui est interne et «l'espace de l'autre», qui est externe. Bien que ces deux espaces puissent se situer dans le même espace-territoire, ils demeurent des espaces-identités différents, chacun se jouant sur une notion d'extériorité et d'intériorité, d'exclusion et d'inclusion. Le même espace-territoire peut être extérieur et intérieur tout à la fois. Il y a une "frontière» entre les deux et il va sans dire que l'interprétation d'un texte varie selon la perspective de celui qui l'interprète, selon le point de vue de son adhésion à un espaceidentité propre.

Cette opposition peut aussi se jouer sur un plan plus concret, soit entre le centre et la périphérie, entre le haut et le bas. Selon la perspective de celui qui se croit à l'intérieur de l'espace-identité, la perspective change. Si le siège du pouvoir de cet espace-identité se trouve au centre, "l'autre» se trouve dès lors en périphérie. Sur un plan "vertical» et de manière imagée, l'espace-identité de «l'autre" se trouve ainsi dans les greniers et dans les caves, dans des lieux souterrains ou dans le métro. Ces espaces souterrains occupent une place prépondérante et signifiante dans Jésus de Montréal. La frontière entre les deux espaces-identités, un lieu de rencontre ou de séparation selon la perspective dans laquelle le sujet se place, se trouve représentée partout dans le film par des escaliers, des terrasses, des balcons ou des couloirs.

Cette notion de frontière est très importante dans le contexte canadien, comme le souligne de manière éclairante pour notre propos Randy William Widdis :

Il est connu que l'identité et le lieu - deux concepts inter-reliés - sont définis par les frontières et les régions limitrophes. Cependant, ces deux concepts constituent sur les deux plans, un paradoxe qui complique l'identité canadienne. D'une part, les frontières divisent; d'autre part,

'Iouri Lotman, Universe of the mind A semiotic theory of culture, Londres et New York, I.B. Tauris \& Co., 1990, pp. 131-142. 
les zones limitrophes favorisent les échanges menant à des relations fonctionnelless.

Le dernier et long discours de Daniel, dans Jésus de Montréal, semble justement faire allusion à ces types d'éléments qui impliquent un conflit réel ou en puissance entre les espaces-territoires ou les espaces-identités : «Quand vous verrez l'abomination de la désolation, si vous êtes dans les plaines, il faut vous enfuir dans les montagnes. Si vous êtes sur la terrasse, rentrez pas dans la maison prendre vos affaires. Si vous êtes sur la route, retournez pas chez vous»".

Cette idée revient dans de nombreuses scènes-clés. La première scène qui se joue à l'extérieur a lieu dans un espace-frontière, soit celui de l'esplanade devant l'Université de Montréal. Daniel discute avec un professeur de théologie des théories de réinterprétation des textes anciens et des nouvelles connaissances sur Jésus-Christ. Cependant l'archevêché, qui contrôle les finances de la Faculté de théologie, empêche les théologiens de s'exprimer publiquement. Dans cette scène, l'esplanade symbolise un espace-frontière entre l'espace-identité du théologien - qui n'est pas libre, étant dominé par l'archevêché -, et celui du comédien Daniel qui est libre, selon la perspective du théologien. Le théologien craint d'avoir trop parlé : «Mentionnez jamais mon nom, par exemple, s'il vous plait. Vous pourriez me causer des ennuis sérieux. Vous, vous êtes comédien, vous pouvez dire n'importe quoi»».

La scène après le jugement de Daniel, dans un couloir à l'entrée d'un restaurant (au nom ironique dans ce contexte de frontières : "Chez Charon $)^{*}$ ), constitue un deuxième indice du dédoublement entre la liberté et la soumission. Richard Cardinal, avocat et agent

\footnotetext{
${ }^{5}$ Randy William Widdis, «Borders, borderlands and Canadian identity. A Canadian perspectiven, Reoue internationale d'études canadiennes, vol. 15, printemps 1997, p. 49.

'Denys Arcand, Jésus de Montréal, Montréal, Boréal, 1989, p. 175.

'Ibid, p. 35.

${ }^{8}$ Dans la mythologie grecque et romaine, Charon, fils d'Érèbe et de la nuit, reçoit les âmes des morts et leur fait traverser l'Acheron, le fleuve des enfers, au prix d'une obole. Cette frontière est ici identifiée au restaurant où Richard Cardinal (au nom vertueux, mais représentant Satan) tente de pervertir Daniel au jeu de l'argent et du pouvoir.
} 
dans le milieu artistique, discute avec Daniel. Il veut réinterpréter la vie de Daniel en lui inventant une adolescence de marginal qui servira à lui créer un passé conforme à l'idée que l'on se fait d'un acteur, afin de l'intégrer dans l'espace médiatique et commercial. On veut le déplacer de sa position ufrontalière» pour le ramener dans l'espacegiron, c'est-à-dire dans l'espace-territoire, l'espace-identité de ceux qui détiennent le pouvoir. Gilles Marsolais interprète cette séquence qui symbolise la tentation de Satan : «[...] Cardinal, l'agent artistique, tente de corrompre Daniel en lui faisant miroiter la possibilité de passer dans l'autre classe sociale, dans la classe de ceux qui dominent la Citér».

\section{Le monde : une vision du Canada}

À la lumière de ces diverses propositions, on peut se demander si Jésus de Montréal peut être interprété selon des notions de perception d'espace-identité par opposition à la perception d'espace-territoire. Est-ce que le Canada est un espace-territoire où se jouent de multiples espaces-identités? Est-ce que la structure du Canada repose sur une grille d'interprétation fixe du passé, structure bipolaire où l'espace-interprétation est dominé par le pouvoir majoritaire, ou bien sur une grille d'interprétation plus flexible, basée sur une notion d'identité ou de perception de ces identités plus autonomes et provisoires? La situation conflictuelle associée à la bipolarisation a été évoquée par Réjean Pelletier lors du colloque "Politiques des territoires", qui s'est tenu à Bordeaux en 1994. Il explique à cet égard :

Les conflits deviennent nettement plus aigus lorsqu'ils sont ancrés dans une bipolarisation ethnique ou communautaire. Cette troisième condition se définit par la présence sur un même territoire de deux communautés où l'une est majoritaire et veut affirmer son nombre et ses droits de majorité et l'autre est minoritaire et réclame une

${ }^{9}$ Gilles Marsolais, automne 1989, op. cit., p. 42. 
protection spéciale. [...] Cette bipolarisation se retrouve au Canada ${ }^{10}$.

La bipolarisation et la notion de domination sont représentées dans Jésus de Montréal selon un enchaînement d'images d'espaces publics ou privés. Selon l'espace évoqué à l'écran et la perspective de celui ou de ceux qui détiennent le pouvoir sur l'espace, l'interprétation des thèmes de ce film change. On y retrouve de nombreux types d'espaces, depuis l'espace théâtral et filmique jusqu'à l'espace physique - paysage urbain, jardins, panoramas -, en passant par «l'Espace» en tant que tel - celui du néant qui a vu la création du monde, ou bien l'espace abstrait, celui de l'interprétation.

Nous avons cité plus haut la division proposée par Lotman entre extérieur et intérieur. Cette opposition peut aussi être interprétée dans la perspective d'ordre et de désordre. L'un des exemples étudiés par Lotman, tiré du Décaméron, propose une application de cette notion. Selon Lotman, ce livre peut être divisé selon une opposition entre paysage (régi par des connotations de paix, le jardin et locus amœnus) et ville, qui signifie désordre et violence". Bien que le film de Denys Arcand ne soit pas structuré selon une opposition entre paysage et ville, introduisant les connotations citées, d'autres divisions existent entre intérieur et extérieur, parc (ou jardin) et ville, qui jouent un rôle important dans une scène qui sera analysée plus tard.

Sur un plan sociopolitique, David $\mathbf{H}$. Kaplan a proposé des modèles spatiaux de ce messagen. Il examine les tensions qui émergent lorsqu'un groupe, à l'intérieur d'une nation, a le sentiment que son identité spatiale ne coïncide pas avec l'identité spatiale accordée par le groupe qui détient le pouvoir. Nous allons voir

${ }^{10}$ Réjean Pelletier, sLe Québec et le Canada. Asymétrie des pouvoirs et logique d'égalitén, J.P. Augustin [éd.], L'Institutionnalisation du territoire au Canada, Québec, Les Presses de l'Université Laval et Bordeaux, Presses universitaires de Bordeaux, 1996, p. 172.

"Iouri Lotman, op. cit., pp. 131-142.

1 David H. Kaplan, Two nations in search of a state. Canada's ambivaent spatial identities", Annals of the Association of American Geographers, vol. 84, $n^{\circ} 4,1994$, pp. 585606. 
comment ces notions sont applicables au Québec, application qui pourrait aussi sans aucun doute être valide de manière moins particulière.

Dans le film qui nous occupe ici, l'espace extérieur domine. Bien que, sur les 115 minutes du film, seulement les deux cinquièmes environ se passent à l'extérieur, un lien avec le monde extérieur est souvent représenté par un espace-frontière. On pourrait citer en guise d'exemple la mise en scène souterraine de la mort de Daniel dans le métro, scène qui suit la dernière représentation extérieure de la Crucifixion, où l'accident a eu lieu. On y voit les escaliers roulants qui descendent et qui montent, les trains qui arrivent, symboles de liens avec le monde de l'extérieur. De même, le décor des scènes qui se jouent à l'intérieur des bâtiments s'accompagne le plus souvent de plantes, évoquant ouvertement l'espace du jardin, lequel est aussi suggéré par le moyen de fenêtres qui donnent sur la ville.

L'importance du jardin est dans ce film primordiale. Le «jardin" est le parc qui entoure l'oratoire Saint-Joseph du mont Royal. La fiction du parc du sanctuaire se joue à côté du vrai parc, les deux environnements ayant leurs propres champs de signification. Le jardin lié à l'intrigue de Jésus de Montréal évoque pour le public occidental la Bible et surtout le jardin d'Éden d'où l'homme est chassé. Prenons par exemple le dialogue qui a lieu vers la fin du film entre les comédiens et Bob Chalifoux, garde de sécurité du sanctuaire. Les comédiens viennent alors d'apprendre qu'il ne pourront plus jouer la pièce :

CONSTANCE Non, mais on pourrait jouer dans un parc.

MARTIN On n'obtiendra pas l'autorisation.

MIREILLE Il y a pas des jardins quelque part?

RENE C'est toujours privé'.

Les comédiens n'auront donc jamais l'autorisation de jouer dans un parc et, quant aux jardins, bien qu' «ouverts» à tous, ils sont toujours privés. Ce sont des espaces-territoires qui ont été dressés en

${ }^{\mathfrak{1}}$ Denys Arcand, op. cit., pp. 158-159. 
espaces-frontières, empêchant de la sorte quiconque de menacer la différence leur «identité». La seule solution envisageable semble être un changement d'identité, c'est-à-dire la modification du spectacle de la Passion en vue de le rendre conforme aux normes ecclésiastiques. Le père Leclerc explique aux comédiens que les autorités du sanctuaire, qui ont vu la représentation, exigent des modifications considérables. Il n'y aurait pas de problèmes, dit-il, si les comédiens voulaient jouer la pièce dans un théâtre, dans la ville, «mais vous êtes ici dans les jardins privés d'un sanctuaire [pause] catholique. Ça impose un certain nombre de restrictions ${ }^{4}$.» Il propose un compromis, soit de continuer à jouer la pièce, mais en reprenant les anciens textes qu'on jouait les années précédentes. Les comédiens refusent d'accepter ce compromis et de changer l'identité de la pièce, et ils continuent leur répétition.

Cette répétition reprend le concept de l'espace théâtral tel qu'on le voyait au début du film, mais de manière différente et beaucoup plus complexe. Elle a lieu dans le jardin avec pour seuls spectateurs le père Leclerc et, derrière celui-ci et à son insu, Daniel Coulombe qui revient vers sa troupe, comme si les espaces avaient éclaté. Il n'y a pas d'autre public pour voir la répétition. Il y a certes des gens qui regardent, mais ce sont les comédiens qui jouent en même temps, traversant ainsi la frontière entre les espaces-territoires. Leclerc joue son rôle de gardien des lois et des règlements dans son propre espaceterritoire extérieur à celui des comédiens, mais il est aussi spectateur au sein de l'espace-territoire des comédiens. Il agit, écoute et voit ce qui se passe tout à la fois. D'abord sur le balcon-estrade au même niveau que les comédiens, puis, après en avoir descendu les marches, sur la pelouse dans son espace propre. Le jardin représente donc pour lui un espace inférieur, tandis que les acteurs restent à un niveau plus élevé dans l'espace-terrritoire qu'il leur a cédé sur l'estrade de pierre. Si Daniel n'y est pas au début, il arrive par la suite de l'extérieur pour écouter, pour être le témoin de la scène. Il reste derrière Leclerc, qui ne le voit pas, mais il est clairement visible de ses amis. Il fait donc partie du jeu de ceux-ci. Il est symboliquement

"Ibid, p. 148. 
dans leur espace, bien qu'il soit dans le même espace «inférieur» où se trouve Leclerc.

En plein jour, alors que le soleil est encore haut dans le ciel, un grand espace est ouvert, mais Leclerc, symbole de messages fixes et d'interdictions, reste la personne qui croit voir ce qui se passe, mais ne voit pas tout. Leclerc est le "propriétaire» de cet espace-territoire composé du jardin et de l'estrade, mais il ne comprend pas ce qui s'y passe. Sa vision est fermée par ses supérieurs catholiques. L'identité de l'espace lui échappe donc.

Il s'agit là d'une situation doublement paradoxale. Leclerc détient le pouvoir dans son espace-territoire, mais, étant obligé de suivre les ordres de ses supérieurs, il accepte leur identité et perd la sienne, puisqu'il va à l'encontre de ce qu'il prêche depuis le début du film. En même temps, il est physiquement inférieur aux comédiens qui occupent son espace-territoire. Donc, bien qu'il détienne le pouvoir, il a perdu toute autorité. Il est exclu de son propre espaceidentité.

Ce sont maintenant les comédiens qui contrôlent son espace. Leclerc est obligé d'assister à plusieurs interprétations différentes du même texte. Les comédiens jouent de différentes manières son texte plat et sans intérêt (celui qui était joué les années précédentes), en lui donnant des significations plutôt érotiques, dans un style "Comédie française», "method acting» comme à New York's et finalement en langue populaire québécoise. Les comédiens jouent la scène sur un mode ironique. Ils demandent à Leclerc s'il veut qu'ils interprètent le texte de manière moins locale et plus internationale, se lançant alors dans une interprétation japonaise Nô.

Ce faisant, les comédiens font la leçon au père Leclerc. Il est forcé de saisir enfin que n'importe quel texte est ouvert à une multitude d'interprétations. Le texte est comme un espace-territoire qui peut avoir son propre espace-identité, voire ses propres espacesidentités. En entrant dans un jeu de création - de nouvelles significations et de nouvelles identités -, les comédiens ont

${ }^{15} \mathrm{Ibid}, \mathrm{p} .149$. 
transformé le jardin du sanctuaire en un nouveau jardin d'Éden. Est lié à cette interprétation, le texte de l'Évangile selon saint Jean qui, seul parmi les quatre Évangiles, décrit le lieu de crucifixion et le lieu d'enterrement de Christ comme un jardin's. La mort du Christ a donc lieu dans un jardin selon l'un des Évangiles, et pas selon les trois autres. Les «anciens textes» varient donc, tandis que les autorités catholiques veulent les réduire à un seul sens.

\section{Le Québec, Montréal et les Montréalais : des visions de l'autre}

Cette analyse des divisions des espaces touche très spécifiquement le Québec. Un tout autre champ de signification apparaît lorsqu'on adjoint à ces interprétations celles qui pourraient être faites par un public connaissant Montréal. L'oratoire sur le mont Royal est reconnu comme un des lieux de recueillement spirituel les plus importants au monde. Le mont Royal lui-même s'offre comme le noyau sémantique de la ville de Montréal ${ }^{p}$, aussi bien que comme son centre visuel. En ce sens, le mont Royal représente pour les individus qui le connaissent un lieu de significations historiques et religieuses du Québec, connaissances qui s'ajoutent aux significations bibliques et mythologiques du jardin. Le noyau créatif du film se divise ainsi en devenant particulier à chaque individu selon son espace-identité ou son espace-territoire, peu importe qu'il connaisse la Bible en profondeur, en autant qu'il en connaisse les grandes lignes ou qu'il soit de Montréal.

Il existe donc un lien étroit entre la fiction du film et la réalité quotidienne de la vie au Québec. Arcand a en effet suggéré à cet égard :

Nous [les documentaristes] notre référence principale, c'est le coin de la rue. Nous nous sentons limités par la

\footnotetext{
${ }^{16}$ Evangile selon saint Jean, 19,41.

${ }^{17}$ Puisque Montréal (mont Royal) en tire son nom.
} 


\section{REVUE INTERNATIONALE D'ÉTUDES QUÉBÉCOISES}

réalité, alors que la fiction n'est pas la réalité. La fiction, c'est une invention qui doit être tellement géniale qu'elle arrive à rejoindre la réalité par l'autre sphère de la création ${ }^{18}$.

Jésus de Montréal explore la réalité au moyen de l'espace entre le documentaire et la fiction en se servant de modèles d'espaces pour le jeu des interprétations. Les espaces - surtout celui du jardin qu'Arcand nous offre sous forme de documentaire, donnent son impulsion à la fiction. Ce qu'ils représentent joue un rôle prépondérant dans l'interprétation du film.

Selon Kaplan cité ci-dessus", le Canada a toujours maintenu deux identités distinctes et plutôt fixes, l'identité canadienne-anglaise et l'identité canadienne-française autrefois, aujourd'hui l'identité québécoise. Il propose des modèles qui indiquent les différences dans la perception de ces identités. Il fait une comparaison entre la perception française de l'identité et la perception anglaise en 1900 et en 1994 à l'intérieur d'un même et seul pays. En 1900, l'identité «française" se maintenait comme espace-territoire à une échelle très localisée au Québec, sous l'égide de l'Église catholique, au sein d'un réseau de "paroisses nationales", à l'intérieur d'un espace perçu comme un espace-identité continental ${ }^{20}$. À la même époque, les Canadiens anglais percevaient leur espace-territoire comme plus vaste, comprenant le territoire où se trouvaient les "paroisses nationales». Pour les Canadiens anglais, l'espace-identité occupait non seulement le continent mais aussi la Grande-Bretagne. Kaplan montre ensuite le renversement de cette situation vers 1994 , en mettant au jour les différences survenues à la suite de la Révolution tranquille au Québec, différences qui se donnaient à voir surtout dans le mouvement des provinces vers le Québec et le changement d'une identité rurale dispersée vers une identité urbaine plus centralisée. Ce mouvement s'est renforcé avec l'arrivée au pouvoir du Parti québécois en 1976, qui a réussi à donner au Québec une véritable influence politique. On pourrait proposer l'hypothèse

\footnotetext{
18 Marcel Jean, «Entretien avec Denys Arcand», 24 Images, ns 44-45, automne 1989, p. 52.

${ }^{19}$ David H. Kaplan, op. cit., pp. 585-606.

${ }^{20}$ Ibid, p. 593.
} 
qu'aujourd'hui, les Québécois se perçoivent comme étant au pouvoir au centre, au Québec. Il y aurait dès lors coïncidence entre leur espace-identité et leur espace-territoire, tels que symbolisés dans le film par le jardin qui exclut «l'autre».

Néanmoins, un paradoxe résiste à cette proposition. L'hypothèse formulée de la sorte renforcerait l'idée d'une seule et unique identité, à l'instar de celle prônée par l'Église catholique dans le film. Cependant, nous avons montré la multiplicité d'identités proposées par le jeu des comédiens devant Leclerc.

Si l'on reprend l'image de l'identité spatiale franco-canadienne de 1900 proposée par Kaplan, il y a une autre coïncidence intéressante à la fin de ce film, coïncidence qui offre une interprétation différente. Le jardin du sanctuaire est le lieu où tout pourrait recommencer, une espèce de nouveau jardin d'Éden. Pendant la répétition, un seul espace régi par deux identités distinctes commence à se séparer pour leur permettre de coexister l'une à côté de l'autre. C'est le modèle que nous propose Kaplan pour 1900: ces identités existent de manière autonome à l'intérieur de Montréal, à l'intérieur du Québec, et par conséquent du Canada.

La conclusion qu'on peut tirer de Jésus de Montréal demeure donc ambiguë. À la fin du film, le corps de Daniel est dispersé pour redonner vie à des êtres différents. Denys Arcand affirme à ce sujet que [....] le fait que ses organes soient redistribués dans divers hôpitaux pour guérir ou redonner vie à des patients indique [...] qu'il n'est pas mort en vain, malgré les apparences. ${ }^{\eta_{\text {}}}$ On pourrait certes dire qu'il a été assimilé, qu'il n'existe plus comme identité, mais on pourrait aussi ajouter que sa mort a donné vie à d'autres identités, à des corps individuels qui occupent l'espace de base, l'espace minimum de d'autres identités, d'autres territoires.

Cependant, malgré tout ce qu'il a accompli, Daniel meurt à la fin, dans une mort annoncée à la fin de la répétition où il faisait semblant de se faire hara-kiri. Satan, sous la forme de Cardinal, est toujours vivant à la fin du film, et il tente cette fois les comédiens en leur

${ }^{2}$ Gilles Marsolais et Claude Racine, op. cit., p. 9. 
offrant un projet de contrat. Ces éléments soulignent, de leur côté, le symbolisme et l'ironie des parallèles entrevus avec le jardin d'Éden.

On pourrait dès lors suggérer que le jeu des espaces dans ce film coïncide avec les débats autour des espaces-identités et des espacesterritoires au Québec et au Canada. Ces débats touchent non seulement les groupes majoritaires, mais aussi les minorités qui, elles aussi, veulent profiter d'une identité à l'intérieur d'un espace. C'est ce que souligne par ailleurs Julien Bauer :

Si les politiques visant spécifiquement les minorités les placent en position d'infériorité, les développements qu'a connus le Québec depuis un quart de siècle ont entraîné des changements considérables et souvent positifs pour ces mêmes minorités. À l'origine les minoritaires étaient marginaux, perçus comme des étrangers, poussés dans les bras des institutions anglaises. Aujourd'hui, ils sont à l'ordre du jour, sont encore perçus comme des immigrants, sont devenus l'enjeu d'une concurrence entre les deux groupes linguistiques; ils sont de plus en plus présents et indispensables à l'épanouissement du Québec. Les «autres» représentaient un danger. Ils sont devenus un atout ${ }^{2}$.

Tout porte à croire qu'il s'agit là de la raison pour laquelle ce film a obtenu un succès retentissant. L'ambiguïté du discours dans le film en fait sa grande force. Par le jeu des espaces différents, chacun peut y lire un message qui renforce sa propre notion de territoire et d'identité. Chacun peut espérer y trouver un espace-territoire où il peut jouir de son espace-identité.

" Julien Bauer, Les Minorités au Québec, Montréal, Boréal, 1994, p. 118. 